\title{
Variations in Metformin Prescribing for Type 2 Diabetes
}

\author{
Tiffany Goldberg, PharmD, Miranda E. Kroehl, MS, PhD, \\ Kathleen Heist Suddarth, MD, and Katy E. Trinkley, PharmD
}

Background: Reasons for suboptimal metformin prescribing are unclear, but may be due to perceived risk of lactic acidosis. The purpose of this study is to describe provider attitudes regarding metformin prescribing in various patient situations.

Methods: An anonymous, electronic survey was distributed electronically to 76 health care providers across the nation. The 14-item survey contained demographic questions and questions related to prescribing of metformin for T2DM in various patient situations, including suboptimal glycemic control, alcohol use, history of lactic acidosis, and varying degrees of severity for certain health conditions, including renal and hepatic dysfunction, chronic obstructive pulmonary disease, and heart failure.

Results: There were a total of 100 respondents. For suboptimal glycemic control, most providers (75\%) would increase metformin from 1500 to $2000 \mathrm{mg}$ daily; however, $25 \%$ would add an alternate agent, such as a sulfonylurea (18\%) or dipeptidyl peptidase-4 inhibitor (7\%). Although $51 \%$ of providers would stop metformin based on serum creatinine thresholds, the remainder would rely on glomerular filtration rate thresholds of $<60 \mathrm{~mL} / \mathrm{min}(15 \%),<30 \mathrm{~mL} / \mathrm{min}(33 \%)$, or $<15 \mathrm{~mL} / \mathrm{min}(1 \%)$ to determine when to stop metformin. For heart failure, $45 \%$ of providers would continue metformin as currently prescribed regardless of severity. Most providers would adjust metformin for varying severity of hepatic dysfunction (74\%) and alcohol abuse (40\%).

Conclusions: Despite evidence supporting the cardiovascular benefits of metformin, provider attitudes toward prescribing metformin are suboptimal in certain patient situations and vary greatly by provider. (J Am Board Fam Med 2015;28:777-784.)

Keywords: Health Care Surveys, Lactic Acidosis, Metformin, Physician's Practice Patterns, Type 2 Diabetes Mellitus

Metformin, is the only medication for type 2 diabetes (T2DM) that has demonstrated reproducible benefits on cardiovascular morbidity and mortality. ${ }^{1}$ Thus, metformin is considered first-line treatment for T2DM per the American Diabetes Association's clinical practice guidelines. ${ }^{2}$ In the UK

This article was externally peer reviewed.

Submitted 13 February 2015; revised 15 May 2015; accepted 1 June 2015.

From the Skaggs School of Pharmacy and Pharmaceutical Sciences, Department of Clinical Pharmacy (TG, KET), the School of Public Health (MEK), and the School of Medicine, Department of Medicine (KHS, KET), University of Colorado, Aurora.

Funding: none.

Conflict of interest: none declared.

Corresponding author: Katy E. Trinkley, PharmD, BCACP, Department of Clinical Pharmacy, University of Colorado Skaggs School of Pharmacy and Pharmaceutical Sciences, Department of Medicine, School of Medicine, $12850 \mathrm{E}$. Montview Blvd, Mailstop C238, Aurora, CO 80045 (E-mail: katy.trinkley@ucdenver.edu).
Prospective Diabetes Study (UKPDS), cardiovascular morbidity and mortality benefits were demonstrated in patients with uncontrolled diabetes who were treated daily with metformin at doses of 1700 to $2550 \mathrm{mg}^{1}{ }^{1}$ At these doses, metformin reduced the risk of any diabetes-related end point, myocardial infarction, and all-cause mortality by $32 \%(P=.017), 39 \%(P=.010)$, and 36\% $(P=$ $.011)$, respectively. ${ }^{1}$ Despite these findings, manufacturer guidelines suggest that the minimum effective dose of metformin is $1500 \mathrm{mg} /$ day. ${ }^{3} \mathrm{In}$ accordance with the manufacturer, other commonly used tertiary references also cite $1500 \mathrm{mg}$ daily as the minimum effective dose of metformin. ${ }^{4,5}$ Despite the clear benefits on glycemic control, morbidity, and mortality, metformin prescribing remains suboptimal. ${ }^{6,7}$ It is estimated that metformin treatment is not initiated in nearly $50 \%$ of patients with T2DM. ${ }^{8}$ 
Reasons for this suboptimal prescribing are not clearly defined in the literature, but they may be attributable to fear of precipitating lactic acidosis in patients with known risk factors. Potential risk factors for lactic acidosis include chronic kidney disease, hepatic dysfunction, heart failure, chronic obstructive pulmonary disease (COPD), alcohol abuse, and a history of lactic acidosis. Although there is little information on the exact risk of lactic acidosis with metformin, data suggest that the association is negligible; one study demonstrated an incidence of 4.3 per 100,000 patient-years. ${ }^{9}$ Furthermore, previous studies demonstrated no increased risk of lactic acidosis with metformin use in the settings of heart failure and renal dysfunction. ${ }^{10-12}$ Despite these data, the manufacturer recommends discontinuing metformin when serum creatinine exceeds $1.5 \mathrm{mg} / \mathrm{dL}$ for men and $1.4 \mathrm{mg} / \mathrm{dL}$ for women, ${ }^{3}$ likely because of concerns for risk of lactic acidosis. This fear of increased lactic acidosis risk with metformin may stem from a preexisting medication, phenformin, which was associated with fatal cases of lactic acidosis in patients and has since been removed from the market. ${ }^{13}$ However, there are few data to support similar concerns with metformin.

There is a need to gain an understanding of the current prescribing practices for metformin to optimize metformin prescribing for T2DM, ${ }^{9}$ such that more patients with diabetes can benefit from metformin's unique cardiovascular morbidity and mortality effects. The purpose of this study was to gain a better understanding of metformin prescribing attitudes among health professionals in various clinical scenarios.

\section{Methods}

A 14-item survey was developed and modified based on feedback from a small focus group of primary care providers, consisting of 1 physician assistant and 4 physicians. During the focus group, cognitive testing of the survey was conducted in which the providers were asked to (1) complete the survey; (2) think aloud about how they constructed their answers; (3) discuss their interpretation of the meaning of each item; (4) report any difficulties in answering the questions; and (5) provide any other feedback. After incorporating the feedback from the pilot, the final survey included the same 14 items with the language modified for clarity. Four questions related to demographical information, including specific profession, duration of practice, practice site, and geographic location of practice. The remaining 10 questions related to prescribing attitudes in various clinical scenarios, including suboptimal glycemic control and presence of risk factors for lactic acidosis, including renal dysfunction, hepatic dysfunction, heart failure, COPD, alcoholism, history of lactic acidosis, and current lactic acidosis.

The survey was anonymous and distributed electronically using the online survey database Qualtrics. Survey participants were provided postcard consent before completing the survey, explaining that they were giving consent to participate by completing the survey. The survey was distributed to a convenience sample of people with ongoing professional relationships with the study investigators, and similar to the methodology of a prior study, recipients of the survey were asked to forward the survey to other clinician colleagues. ${ }^{14}$ Survey respondents were providers who practiced in a setting in which they were involved in prescribing or recommending chronic drug therapy for T2DM, which was listed as inclusion criteria at the top of the electronic survey. To submit the survey, participants were required to complete all questions. This study was deemed exempt by the University of Colorado's institutional review board.

To determine whether there were characteristics associated with responses to the patient situations, the results from each situation were categorized into a binary outcome indicating whether the respondent reported they would take the recommended action or not. For scenarios where more than 1 answer was acceptable, respondents who selected any scenarios not within the suggested guidelines were considered as not following a recommended action. Individual logistic regression models were used to estimate the relationships between profession (physician vs pharmacist, $\mathrm{n}=96$ ), years practicing $(\mathrm{n}=100)$, location (Midwest vs Mountain West, $\mathrm{n}=97$ ), and clinic type (academic vs all other, $\mathrm{n}=100$ ) for each patient situation. Given the small number of responses from the South region and midlevel practitioners, they were not included in the logistic regression models. To account for multiple comparisons, a false discovery rate adjustment was applied to the $P$ values. All 
analyses were conducted using SAS software (SAS Institute, Cary, NC).

\section{Results}

The survey was distributed nationwide to 76 providers who had ongoing professional relationships with the investigators. The survey was open from November 10, 2013, until January 17, 2014. A total of 101 surveys were completed as a result of survey respondents forwarding the survey to their clinician colleagues.

The majority of respondents (66\%) were physicians who completed their residency training, $20 \%$ were pharmacists, and $10 \%$ were medical residents. The remaining $4 \%$ were divided evenly among nurse practitioners and physician assistants. Years of clinical experience were evenly distributed between $<5$ years to $>15$ years of experience. The majority of respondents practiced in the Mountain West region (68\%) and in an academic clinic (61\%). Demographic information, including details of geographic region, is described in Table 1.

Provider attitudes regarding metformin prescribing in various clinical scenarios are described in Table 2. In patients with uncontrolled diabetes and an $\mathrm{A} 1 \mathrm{C}$ of $8.3 \%$, most providers (84\%) chose to titrate metformin from 500 to $2000 \mathrm{mg}$ daily, whereas $11 \%$ chose metformin $1500 \mathrm{mg}$ daily as an appropriate regimen. Interestingly, in a similar patient with an $\mathrm{A} 1 \mathrm{C}$ of $7.3 \%, 75 \%$ of providers would increase metformin from 1500 to $2000 \mathrm{mg}$ daily, whereas $18 \%$ of providers chose to add a sulfonylurea.

Of providers, $50 \%$ followed the metformin manufacturer's recommendations to discontinue metformin use when serum creatinine is $>1.5$ $\mathrm{mg} / \mathrm{dL}$. While the other $50 \%$ of providers accepted glomerular filtration rate (GFR) as a better indicator of when to discontinue metformin use, there seems to be no consensus among providers on what the GFR cutoff should be. In patients taking metformin $1750 \mathrm{mg}$ daily and with either hepatic dysfunction or heart failure, there was great variation in provider prescribing attitudes and no notable trends. For patients with COPD, however, $90 \%$ of providers did not alter the patient's metformin therapy. In patients with alcoholism, 59\% of providers would not alter metformin dosing, but many providers would
Table 1. Demographic Characteristics of Survey Respondents $(\mathrm{n}=100)$

\begin{tabular}{|c|c|}
\hline Demographic Information & Respondents (\%) \\
\hline \multicolumn{2}{|l|}{ Clinical profession } \\
\hline Medical resident & 10 \\
\hline Physician, completed residency training & 67 \\
\hline Nurse practitioner & 2 \\
\hline Physician assistant & 2 \\
\hline Pharmacist & 19 \\
\hline \multicolumn{2}{|l|}{ Years practicing in clinical profession } \\
\hline$<5$ years & 31 \\
\hline $5-10$ years & 24 \\
\hline $11-15$ years & 17 \\
\hline$>15$ years & 28 \\
\hline \multicolumn{2}{|l|}{ Geographic location } \\
\hline $\begin{array}{l}\text { Midwest (IL, IN, IA, KS, MI, MN, MO, } \\
\text { NE, ND, OH, OK, SD, WI) }\end{array}$ & 29 \\
\hline $\begin{array}{l}\text { South (AL, AR, FL, GA, KY, LA, MS, } \\
\text { NC, SC, TN, TX, VA, WV) }\end{array}$ & 3 \\
\hline $\begin{array}{l}\text { Mountain West (AZ, CO, NV, NM, } \\
\text { UT, WY) }\end{array}$ & 68 \\
\hline \multicolumn{2}{|l|}{ Type of clinic } \\
\hline Federally qualified healthcare clinic & 17 \\
\hline Academic clinic & 61 \\
\hline Private clinic & 5 \\
\hline County hospital clinic & 7 \\
\hline Veterans affairs clinic & 3 \\
\hline Other & 7 \\
\hline
\end{tabular}

based on varying degrees of alcohol use. For patients with current or a history of lactic acidosis, provider attitudes were inconsistent.

There were no strong associations between any of the survey respondent characteristics and following the recommended practice in the patient situations. Results of the logistic regression analyses are shown in Table 3. Physicians were more likely than pharmacists to follow the suggested practices for 2 of the 10 situations presented, and those working in an academic setting were more likely than those working in any other setting to follow the suggested practices for 3 of the situations; however, statistical significance was lost after applying the false discovery rate adjustment.

\section{Discussion}

These findings suggest that attitudes toward metformin prescribing vary significantly among providers. There seems to be little consensus among providers in how to adjust metformin prescribing based on glycemic control or comorbidities. To 
Table 2. Provider $(n=100)$ Attitudes Toward Prescribing Metformin in Various Clinical Situations

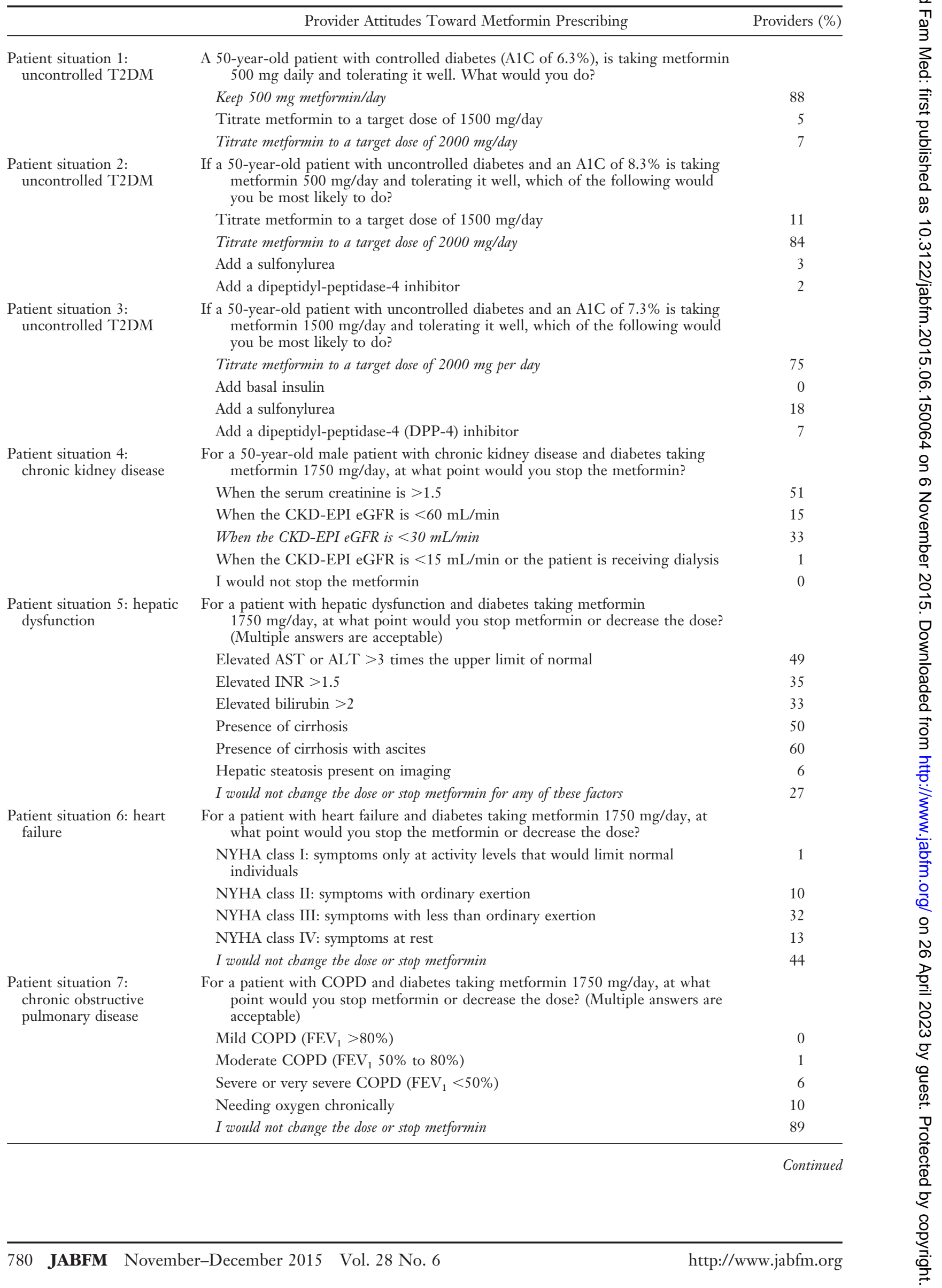


Patient situation 8: alcohol abuse

Patient situation 9: history of lactic acidosis

Patient situation 10 : current lactic acidosis
For a patient with alcoholism and diabetes taking metformin $1750 \mathrm{mg} /$ day, at what point would you stop metformin or decrease the dose? (Multiple answers are acceptable)

If they are dependent on alcohol 28

If they abuse alcohol 29

If they consume fewer than 2 drinks/day for men and 1 drink/day for 2 women/elderly

If they consume $>4$ drinks/day or 14 drinks/week, regardless of sex or age 25

I would not change the dose or stop metformin

For a 50 -year-old patient with an A1C of $8.3 \%$ who is not currently taking any diabetes medications, has a remote history of lactic acidosis, and has no other risk factors for lactic acidosis, which one of the following would you do? (Multiple answers are acceptable)

Not start metformin $\quad 18$

Start metformin only $\quad 25$

Start metformin and monitor serum lactic acid $\quad 13$

Start metformin at a lower dose than I usually would $\quad 15$

Start metformin at a lower dose than I usually would and monitor serum lactic 26 acid

Start a sulfonylurea instead of metformin $\quad 29$

Start a diabetes medication other than a sulfonylurea or metformin $\quad 12$

For a 50-year-old patient with an $\mathrm{A1C}$ of $6.3 \%$, who is only taking metformin $1750 \mathrm{mg} / \mathrm{day}$ for diabetes and who has a new diagnosis of lactic acidosis, which one of the following would you do acutely? The patient is not going to be admitted to the hospital, and their glycemia remains normal. (Multiple answers are acceptable)

Stop metformin only

Stop metformin and switch to a sulfonylurea

Stop metformin and switch to a diabetes medication other than a sulfonylurea or metformin

Lower the metformin dose

Lower the metformin dose and monitor serum lactic acid

Continue metformin

Continue metformin and monitor serum lactic acid

\footnotetext{
*Suggested answers are set in italics. For some questions, more than one answer was suggested, given that the available evidence does not suggest one correct answer.

ALT, alanine aminotransferase; AST, aspartate aminotransferase; CKD-EPI, Chronic Kidney Disease Epidemiology Collaboration Equation; COPD, chronic obstructive pulmonary disease; eGFR, estimate glomerular filtration rate; $\mathrm{FEV}_{1}$, forced expiratory volume in 1 second; INR, international normalized ratio; NYHA, New York Heart Association; T2DM, type 2 diabetes mellitus.
}

achieve cardiovascular morbidity and mortality benefits, the best available evidence suggests targeting metformin doses of 1700 to $2550 \mathrm{mg}$ daily. However, $11 \%$ of providers still chose to titrate to metformin $1500 \mathrm{mg}$ daily versus 2000 $\mathrm{mg}$ daily in an uncontrolled diabetic patient currently taking metformin $500 \mathrm{mg}$ daily. This is likely because of the metformin manufacturer's recommendations for the minimum effective dose. ${ }^{3}$ What is less clear from UKPDS is whether patients with controlled T2DM would benefit from escalating doses of metformin to the target range of 1700 to $2550 \mathrm{mg}$ daily, given that sub- jects in UKPDS had uncontrolled T2DM. The survey results demonstrate that the majority of providers would not escalate metformin to the target range of 1700 to $2550 \mathrm{mg}$ daily for patients with controlled T2DM.

While $50 \%$ of providers followed the metformin package insert recommendations to discontinue metformin when serum creatinine was $>1.5 \mathrm{mg} / \mathrm{dL}$ for men, the remainder relied on GFR as a better indicator for discontinuing metformin use. Since there are no clear recommendations from the manufacturer regarding GFR cutoffs, providers seem to be split on when it is appropriate to discontinue 
Table 3. Provider Characteristics and Associations with Prescribing Metformin in Various Clinical Situations

\begin{tabular}{|c|c|c|c|c|c|}
\hline Provider Characteristics & Patient Scenario & $\begin{array}{l}\text { Odds } \\
\text { Ratio }\end{array}$ & $95 \% \mathrm{CI}$ & $P$ Value & $\begin{array}{c}\text { FDR-Adjusted } \\
P \text { Value }\end{array}$ \\
\hline \multirow[t]{10}{*}{ Physicians* vs Pharmacists } & 1. Controlled T2DM & 4.4 & $0.6-33.6$ & .1517 & .4727 \\
\hline & 2. Uncontrolled T2DM, metformin $500 \mathrm{mg} /$ day & 0.9 & $0.2-3.6$ & .9088 & .9729 \\
\hline & 3. Uncontrolled T2DM, metformin $1500 \mathrm{mg}$ /day & 1.5 & $0.5-4.6$ & .4614 & .8057 \\
\hline & 4. Chronic kidney disease & 1.2 & $0.4-3.4$ & .7746 & .9683 \\
\hline & 5. Hepatic dysfunction & 1.0 & $0.3-3.3$ & .9335 & .9729 \\
\hline & 6. Heart failure & 2.5 & $0.8-7.5$ & .1138 & .4270 \\
\hline & 7. Chronic obstructive pulmonary disease & 8.4 & $2.1-34$ & .0028 & .1108 \\
\hline & 8. Alcohol abuse & 3.4 & $1.2-9.6$ & .0229 & .2663 \\
\hline & 9. History of lactic acidosis & 1.4 & $0.5-4.4$ & .5353 & .8236 \\
\hline & 10. Current lactic acidosis & 1.5 & $0.5-4.1$ & .4438 & .8057 \\
\hline \multirow[t]{10}{*}{ Years practicing } & 1. Controlled T2DM & 1.7 & $0.7-4$ & .2527 & .7221 \\
\hline & 2. Uncontrolled T2DM, metformin $500 \mathrm{mg} /$ day & 0.6 & $0.4-1$ & .0399 & .2663 \\
\hline & 3. Uncontrolled T2DM, metformin $1500 \mathrm{mg}$ /day & 1.1 & $0.8-1.7$ & .4990 & .8057 \\
\hline & 4. Chronic kidney disease & 0.7 & $0.5-1.1$ & .1174 & .4270 \\
\hline & 5. Hepatic dysfunction & 1.0 & $0.7-1.5$ & .9878 & .9878 \\
\hline & 6. Heart failure & 1.0 & $0.7-1.4$ & .9354 & .9729 \\
\hline & 7. Chronic obstructive pulmonary disease & 1.0 & $0.6-1.8$ & .8681 & .9729 \\
\hline & 8. Alcohol abuse & 1.1 & $0.8-1.5$ & .7582 & .9683 \\
\hline & 9. History of lactic acidosis & 1.1 & $0.7-1.5$ & .7199 & .9635 \\
\hline & 10. Current lactic acidosis & 1.3 & $0.9-1.8$ & .1166 & .4270 \\
\hline \multirow{10}{*}{$\begin{array}{l}\text { Mountain West vs } \\
\text { Midwest region }\end{array}$} & 1. Controlled T2DM & 1.6 & $0.3-10.2$ & .6152 & .8789 \\
\hline & 2. Uncontrolled T2DM, metformin $500 \mathrm{mg} /$ day & 1.7 & $0.5-5.3$ & .3562 & .8057 \\
\hline & 3. Uncontrolled T2DM, metformin $1500 \mathrm{mg} /$ day & 1.0 & $0.4-2.9$ & .9484 & .9729 \\
\hline & 4. Chronic kidney disease & 2.4 & $0.9-6.6$ & .0978 & .4270 \\
\hline & 5. Hepatic dysfunction & 0.7 & $0.3-1.8$ & .4404 & .8057 \\
\hline & 6. Heart failure & 1.0 & $0.4-2.3$ & .9486 & .9729 \\
\hline & 7. Chronic obstructive pulmonary disease & 2.6 & $0.7-9.9$ & .1536 & .4727 \\
\hline & 8. Alcohol abuse & 0.9 & $0.4-2.3$ & .8698 & .9729 \\
\hline & 9. History of lactic acidosis & 1.7 & $0.6-4.6$ & .2838 & .7568 \\
\hline & 10. Current lactic acidosis & 0.7 & $0.3-1.7$ & .4651 & .8057 \\
\hline \multirow{10}{*}{$\begin{array}{l}\text { Academic vs all other } \\
\text { settings }\end{array}$} & 1. Controlled T2DM & 0.4 & $0-3.5$ & .3886 & .8057 \\
\hline & 2. Uncontrolled T2DM, metformin $500 \mathrm{mg} / \mathrm{day}$ & 0.3 & $0.1-1.2$ & .0819 & .4270 \\
\hline & 3. Uncontrolled T2DM, metformin $1500 \mathrm{mg}$ /day & 0.8 & $0.3-2.2$ & .7227 & .9635 \\
\hline & 4. Chronic kidney disease & 3.4 & $1.3-8.9$ & .0128 & .2557 \\
\hline & 5. Hepatic dysfunction & 1.3 & $0.5-3.3$ & .5946 & .8789 \\
\hline & 6. Heart failure & 2.5 & $1.1-5.8$ & .0351 & .2663 \\
\hline & 7. Chronic obstructive pulmonary disease & 0.6 & $0.1-2.2$ & .4032 & .8057 \\
\hline & 8. Alcohol abuse & 0.8 & $0.3-1.7$ & .5036 & .8057 \\
\hline & 9. History of lactic acidosis & 1.5 & $0.6-3.7$ & .3557 & .8057 \\
\hline & 10. Current lactic acidosis & 2.4 & $1.1-5.5$ & .0379 & .2663 \\
\hline
\end{tabular}

*Physicians include residents and those who have completed residency training.

CI, confidence interval; FDR, false discovery rate; T2DM, type 2 diabetes mellitus.

metformin use. There are several major guidelines outside the United States that indicate that GFR is a better measure of kidney function than serum creatinine and should be used to assess metformin use in patients with diabetes. ${ }^{15}$ Although creatinine clearance (using the Cockcroft-Gault equation) is typically preferred for renal drug dose adjustments, metformin dose adjustments have not been evaluated in the same fashion as most other medications; thus GFR, which is a more accurate indicator of renal function, is preferred when dosing metformin. ${ }^{2,13,16}$ While the majority of clinicians would stop metformin because of impaired renal function, it is unclear whether the reason is due to a per- 
ceived risk of lactic acidosis or an alternate reason, perhaps such as fear of legal liability, the contraindication in the manufacturer's package insert, or unfamiliarity with the actual consequences of metformin use.

With regard to metformin use in patients with other comorbidities, such as hepatic dysfunction, heart failure, alcoholism, or history of lactic acidosis, responses indicate there is no consensus on when to adjust metformin therapy, but that the majority of clinicians would alter metformin therapy in varying stages or severities of these conditions. In contrast to renal dysfunction, metformin is not contraindicated in the setting of hepatic dysfunction, heart failure, alcoholism, or history of lactic acidosis; rather, these are considered warnings or precautions to metformin use. ${ }^{3}$ Because these conditions are not contraindications, this may explain the greater variation in responses among providers in contrast to renal dysfunction, in which all clinicians would stop metformin at some degree of renal dysfunction. Nevertheless, the labeling of these comorbidities as warnings or precautions by the drug manufacturer may influence providers' decisions to prescribe metformin. Although many of these clinical situations are fairly common among patients, there are few studies indicating what should be done regarding metformin use in patients with these comorbidities.

Although not statistically significant after adjusting for multiple comparisons, providers practicing in academic clinics were more likely than those in nonacademic settings, and physicians were more likely than pharmacists, to follow the suggested prescribing practices for metformin in certain situations. Future research is needed to better understand these trends.

While this study demonstrated great variation in metformin prescribing, it is limited by the nature of the survey distribution, which was a convenience sample. Using a convenience sample limits the generalizability of the findings. Further, by requesting that respondents forward the survey to their clinician colleagues, we are unable to discern detailed characteristics of the population the survey was distributed to.

Despite the best available evidence suggesting little to no risk of lactic acidosis with metformin use, most clinicians would adjust metformin therapy in the setting of risk factors for lactic acidosis, including renal dysfunction, hepatic dysfunction, heart failure, alcoholism, and history of or current lactic acidosis. However, clinician thresholds for adjusting metformin prescribing are inconsistent. Further research is needed to better define and characterize risk factors for lactic acidosis with metformin use to help inform prescribing of metformin. Additional studies also are needed to better understand why attitudes toward prescribing metformin vary greatly and to allow for targeted interventions, which may be in the form of focused interviews or focus groups to answer these questions. A better understanding of the risk of lactic acidosis with metformin and reasons why prescribing attitudes vary are needed to improve appropriate prescribing of this important diabetes medication. Once more information is known about why providers are reluctant to prescribe metformin in certain clinical situations, implementation of targeted clinical decision support tools, such as electronic alerts within electronic health records, may assist in optimizing the use of this important medication. Further, aligning the manufacturer's recommendations of metformin prescribing with the best available evidence may also assist in optimizing prescribing. Optimizing metformin prescribing is critical to improving cardiovascular morbidity and mortality for the high-risk population of patients with T2DM.

\section{References}

1. Effect of intensive blood-glucose control with metformin on complications in overweight patients with type 2 diabetes (UKPDS 34). UK Prospective Diabetes Study (UKPDS) Group. Lancet 1998;352: 854-65.

2. American Diabetes Association. Standards of medical care in diabetes-2015. Diabetes Care 2015; 38(Suppl 1):S1-90.

3. Glucophage [package insert]. Princeton NJ: Bristol Myers Squibb; 2009.

4. Lexicomp, Lexi-Drugs [database online]. Metformin. Hudson, Ohio: Lexi-Comp, Inc.; January 29, 2015. Available at: www.lexicomponline.com. Accessed October 3, 2015.

5. Drug Facts and Comparisons. Metformin hydrochloride. Facts \& Comparisons [database online]. St. Louis (MO): Wolters Kluwer Health; 2005. Available from: http://online.factsandcomparisons.com. Accessed November 20, 2014.

6. Desai NR, Shrank WH, Fischer MA, et al. Patterns of medication initiation in newly diagnosed diabetes mellitus: quality and cost implications. Am J Med 2012;125:302.e1-7. 
7. Calabrese AT, Coley KC, DuPose SV, Swanson D, Rao RH. Evaluation of prescribing practices: risk of lactic acidosis with metformin therapy. Arch Intern Med 2002;162:434-7.

8. Berkowitz SA, Krumme AA, Avorn J, et al. Initial choice of oral glucose-lowering medication for diabetes mellitus: a patient-centered comparative effectiveness study. JAMA Intern Med 2014;174: $1955-62$.

9. Salpeter SR, Greyber E, Pasternak GA, Salpeter EE. Risk of fatal and nonfatal lactic acidosis with metformin use in type 2 diabetes mellitus. Cochrane Database Syst Rev 2010;(4):CD002967.

10. Norwood DK, Chilipko AA, Amin SM, Macharia D, Still KL. Evaluating the potential benefits of metformin in patients with cardiovascular disease and heart failure. Consult Pharm 2013;28:579-83.

11. Eurich DT, Weir DL, Majumdar SR, et al. Comparative safety and effectiveness of metformin in patients with diabetes mellitus and heart failure: sys- tematic review of observational studies involving 34,000 patients. Circ Heart Fail 2013;6:395-402.

12. Richy FF, Sabido-Espin M, Guedes S, Corvino FA, Gottwald-Hostalek U. Incidence of lactic acidosis in patients with type 2 diabetes with and without renal impairment treated with metformin: a retrospective cohort study. Diabetes Care 2014;37:2291-5.

13. Lipska KJ, Bailey CJ, Inzucchi SE. Use of metformin in the setting of mild-to-moderate renal insufficiency. Diabetes Care 2011;34:1431-7.

14. Maclaren R, McQueen RB, Campbell J. Clinical and financial impact of pharmacy services in the intensive care unit: pharmacist and prescriber perceptions. Pharmacotherapy 2013;33:401-10.

15. Chadban S, Howell M, Twigg S, et al. Assessment of kidney function in type 2 diabetes. Nephrology 2010;15:S146-61.

16. Trinkley KE, Nikels M, Page RL II, Joy MS. Automating and estimating glomerular filtration rate for dosing medications and staging chronic kidney disease. Int J Gen Med. 2014;7:211-8. 